\title{
Prevention of Work-Related Musculoskeletal Disorders in Physiotherapist - A Review
}

\author{
Ruchi G Desai $^{1}$, Manali K Shah ${ }^{2}$ \\ ${ }^{1,2}$ Assistant Professor, L J Institute of Physiotherapy, LJ University, Ahmedabad \\ Corresponding Author: Ruchi G. Desai
}

\begin{abstract}
Introduction: Healthcare professionals, especially those with in direct contact of patient are at the highest risk of work-related musculoskeletal disorders (WMSDs), physical therapists being one of them.

Aim: Our aim was to review current knowledge relating to the prevalence, risk factors and prevention of WMSDs among physiotherapists.

Method: Google Scholar and PubMed were searched for terms relating to WMSDs in Physiotherapists from inception to 2021.

Result: The prevalence of WMSDs among Physiotherapist was high, with the lower back as the most frequently affected followed most often by the neck, upper back and shoulders. Knee joint was commonly affected joint in lower extremity. Multiple risk factors have been found responsible for WMSDs in physiotherapist and some of them are modifiable.
\end{abstract}

Conclusion: Implementing preventive strategies for WMSDs in early stage of a physiotherapist's career may help to prevent WMSDs and maintain good quality of treatment for patients.

Keywords: Pain, Musculoskeletal disorders, Ergonomics, Prevention.

\section{INTRODUCTION}

Physiotherapists are primary health care Professionals who diagnose and treat individuals of all ages, from new-borns with very oldest, who have medical problems or other health related conditions, illness, or injuries that limit their abilities to move and perform the functional activities as well as they would like in their daily lives ${ }^{1}$.
The nature of the work in a physiotherapy practice is physically demanding, and it involves repetitive tasks, high force manual techniques for treating patients, techniques that exert direct pressure on certain joints during treatment, awkward positioning of joints during certain manoeuvres and prolonged constrained postures. ${ }^{1-3}$ These physical factors expose physiotherapists to various work-related musculoskeletal injuries (WMSDs) ${ }^{5}$.

According to World Health Organization (WHO) Musculoskeletal disorders (MSDs) affect the body's muscles, joints, tendons, ligaments and nerves. The term musculoskeletal disorder identifies a large group of conditions that result from traumatizing the body in either a minute or major way over a period of time. Most WMSDs develop over time and are caused either by the work itself or by the employees' working environment. Physical therapists are often at high risk of developing WMSDs as they are mostly involved in physically demanding and intense, repetitive tasks in their practices ${ }^{12}$. The three most important risk factors that have been associated with WMSDs are repetitive tasks, uncomfortable postures and high force levels. Physiotherapists also routinely perform activities such as transferring dependent patients, giving manual therapy, assisting with mat activities, and lifting heavy equipment ${ }^{3}$.

Physiotherapist are reported to be at high risk of WMSDs globally, with the Low back as the major body part affected 
followed by neck and upper back region, shoulder, wrist, knee, thumb and fingers, hip, elbow and legs and toes 4,7,8,9,14. There are multiple risk factors reported by studies to develop work related pain and disorders. It may include numerous risk factors such as repetitive excessive strain on the spine, prolonged awkward static postures (stooping, slouching, ducking), poor positioning, repetitive movements of neck \& shoulders, prolonged application of manual therapy, coping with patient anxietiesmental stress, genetic predisposition, physical conditioning, age, gender, time pressure, high demand \& poor social support etc. ${ }^{7,8,11,20}$

All these factors are responsible for the development of WMSDs over the period of time in physiotherapist. Furthermore, most of the therapists do not take enough break for recovery and most of them take self-treatment or ask a colleague to treat their symptoms instead of taking proper medical advice.

By ignoring symptoms, not taking enough breaks and continuing work without proper medical care may aggravate their condition. Therefore, most of the injured physiotherapists modify their treatment strategies for patient and may change work environment or change the type of patients that they were treating, ultimately reducing their work efficiency towards treating the patients. This all things may lead to disadvantage for patient in form of quality of treatment. Therefore, implementing preventive strategies for WMSDs in early stage of a physiotherapist's career may help to prevent WMSDs and maintain good quality of treatment for patients.

\section{Need of Study}

several studies have assessed the prevalence of WMSDs and its risk factors like gender, age, posture, working hours, work experience etc in physiotherapist. However, studies related to prevalence, risk factors and prevention of WMSDs according to affected body area in physiotherapist is not reviewed. The purpose of study was to review current knowledge associated with prevalence, risk factors, and preventive strategies of WMSDs among physiotherapist according to body area affected.

\section{METHODOLOGY}

Systematic search was done in Google Scholar and PubMed on June 2021 using a predefined strategy for the following key words: work related musculoskeletal disorders, physical therapists, physiotherapists, prevalence, risk factors, and prevention strategies. There were no search limitations. Titles and abstracts of all relevant articles were reviewed. All studies written in English of any design or methodological quality and dealing with WMSDs in PTs were included. Health care professionals other than physiotherapists and articles with unavailable full text were excluded. The reference lists of all the retrieved articles were also searched. Articles and references were analysed logically and conclusion was drawn.

\section{Body}

The lifetime prevalence of WMSDs in many studies was high in physiotherapist. Prevalence in body region most commonly involved are low back, neck, shoulder, upper extremity and lower extremity respectively. This study reviewed region wise prevalence, risk factors and preventive strategies of WMSDs in physiotherapist. Furthermore, in this study relationship between age and gender with WMSDs was reviewed.

\section{Low Back Pain:}

Occurrence of low back pain according to Rugelj D in physiotherapist working in primary health care was $73.7 \%$ and in hospital settings was $50.4 \% .{ }^{15}$ Many other studies showed highest affected area as low back with prevalence of LBP according to Buddhdev \& Kotecha was $35 \%$, Maheshwari et.al was $72.5 \%$ and Malavizhi D et.al was 60\%. ${ }^{3,16,17}$ According to studies common risk factors for LBP are 
treating large number of patients in a single day, bending and twisting the back in awkward position, working in static positions for longer periods, lifting or transferring heavy equipment and dependent patients during sessions, lack of enough rest or break during the day.

It is not possible to eliminate/prevent WMSD because they are multi-factorial, and some risks are non-modifiable (e.g., genetics). ${ }^{19}$ Nevertheless, we can reduce low back pain by minimizing exposure to modifiable risk factors such as awkward postures, heavy physical work, lifting, and forceful movements. ${ }^{18}$ Proper patient handling combined with the use of patient transfer equipment (e.g., lifts), get other personal help for lifting, modifying position of patient or therapist and regular practice of physical activity have been found to reduce Low back pain in healthy professionals. Preventive strategies during activities such as manual therapy, manually resisted exercises and assisted stretches, adequate body mechanics as well as other strategies like change in posture, adjust bed height, use of aids and equipment, using a different body part and substituting electro therapy etc. are needed to minimize load. ${ }^{6,11}$ To reduce the burden of workload, physiotherapists could take breaks/recovery time between patients, and arrange patient's appointment alternately between more and less physically demanding patients.

Adequate strength and flexibility of tissues around spine and pelvis allows full normal spinal movement, prevent abnormal forces on the joints and decreases the possibility of injury. Adequate core strength that comes from abdominal and back muscles helps stabilize the spine, allows proper spinal movement, and makes it easier to maintain correct posture. Strong hip and leg muscles are important to perform proper lifting techniques and body mechanics. ${ }^{10}$ Therefore, strengthening exercises of core and lower extremity should be performed to maintain physical fitness of therapist. Stretching will help to improve flexibility. Various self-stretching exercises should be done at regular interval to prevent muscle strain and soreness as well as to prevent WMSDs at low back in future.

\section{Neck, upper back and upper extremity:}

According to the many studies the neck was the second most affected body part in physiotherapist ${ }^{2}$. Prevalence of neck and upper back pain according to Buddhdev \& Kotecha were $25 \%$ and $15 \%$, Maheshwari et.al were $72.5 \%$ and $28.6 \%$ and Malavizhi D et.al were $62.9 \%$ and $51.9 \%$ respectively. involvement of other joints of upper limb were shoulder, wrist /hand and elbow respectively. 3,16,17 Common WRMDs seen in neck and upper limb in physiotherapist were tension neck syndrome, muscle strain, myalgia, carpal tunnel syndrome, de Quervain's diseases, trigger finger, digital neuritis, carpometacarpal joint arthritis etc. ${ }^{2}$ The potential risk factors of WMSDs for neck, upper back and upper-limb were related to mobilization and manipulation techniques ,therapeutic massage techniques and other hands-on treatment as well as repeatedly performing same task may also lead to such symptoms ${ }^{2}$.Studies also suggested that treating large number of patients in a day is another risk factor for pain in thumb and hand. For prevention physiotherapist can do job rotation, avoid overloading on any particular anatomical area and by avoiding repetitive action as well as other strategies are needed to minimize load. For example, physical therapists could take breaks/recovery time between patients, and arrange patient's appointment alternately between more and less physically demanding patients, use equipment that isn't too heavy or ergonomically designed equipment that can be used without awkward upper body posture to minimize stresses on the upper extremities and the back. Strengthening and various self-stretching exercises should be done at regular interval to prevent muscle strain and soreness as well as to prevent WMSDs at neck, upper back and upper extremities in future. 


\section{Lower limb:}

The most common body part affected in lower limb is knee joint followed by ankle/foot and hip joint respectively. The prevalence of knee according to Bindu $\mathrm{H}$ et.al was $15 \%$, Maheshwari et.al was $12.1 \%$ and Malavizhi D et.al was $30.4 \% 1,16,17$. The higher prevalence of knee symptoms is among paediatric physiotherapists due to the large amount of time spent in kneeling and crouching $^{2,13}$. kneeling position gives more pressure on knee joint; it can be avoided by taking frequent breaks and sitting on soft /firm surface like mat and use of knee- pads while working for longer than an hour in a day continuously ${ }^{10}$. Apart from paediatric physiotherapy, therapist need to assume variety of position in a day during patient's treatment giving manual therapy, stretching and limb physiotherapy and use of electrotherapeutic modalities. For prevention of knee pain in sitting during patient's treatment, physiotherapist should sit to a more ergonomic position, keeping feet on the floor and back straight. While giving treatment in prolong standing position, therapist should assume various derived positions like, walk standing, stride standing etc and keep shifting weight from one to another limb simultaneously to avoid pressure on knee joints ${ }^{10}$.

\section{Relationship of age and WMSDs in physiotherapists:}

Many studies reported the relationship between age and WMSDs among physiotherapist was significantly higher in younger age. The reason suggested by studies was newly qualified therapists tend to work in clinical areas which are physically demanding with more working hours requirement in an order to give direction to their careers. As well as compared to older physiotherapist, newly qualified physiotherapist may have lack of knowledge regarding various coping strategies for prevention of WMSDs.

\section{Relationship of Gender and WMSDs in physiotherapists:}

According to Nordin NAM et al. the prevalence of WMSDS was significantly higher among female physiotherapist. Many other studies have also shown higher prevalence of WMSDs among female physiotherapists ${ }^{2,5}$ and has been attributed to the facts that females are generally smaller in height and physically weaker than males, which may place them at a disadvantage during care task, particularly when lifting \& transferring patients. ${ }^{3,7,16}$

According to studies the male physiotherapists had more neck, wrist and hand, thumb symptoms than female physiotherapists and it may relate to their greater usage of mobilization \& manipulation techniques ${ }^{2}$.Females are also exposed to pregnancy related stress which commonly affects the lower back region due to changes in the spinal posture and a weakening of the joint structures ${ }^{5}$.To prevent abnormal spinal postures following pregnancy in female physiotherapists, postnatal care and core muscle strengthening can be performed.

\section{CONCLUSION}

Physiotherapy is a health care profession, their service is widely distributed from inpatient rehabilitation in ICUs and hospital to outpatient clinics including musculoskeletal physiotherapy, pediatrics and elderly care, neurological rehabilitation, sports field, home care etc. majority studies have shown higher prevalence of WMSDs in physiotherapist which more common in younger physiotherapist, more in female then in male physiotherapist and most common body area affected being low back followed by upper back, neck and shoulder, wrist and hand and knee respectively .common risk factors for WMSDs for physiotherapist were repetitive excessive strain on the spine, prolonged awkward postures, poor positioning, repetitive movements of neck \& shoulders, prolonged application of manual therapy, inadequate work breaks, physical 
conditioning, age, gender, high demand \& poor social support etc. to reduce incidence of WMSDs physiotherapist should be trained and follow preventive strategies from the beginning of their career like physical therapists could take breaks/recovery time between patients, and arrange patient's appointment alternately between more and less physically demanding patients. Along with this physiotherapist should incorporate general strengthening and flexibility exercise in routine and must follow strategies to prevent WMSDs according to body area affected.

\section{Limitation}

As physiotherapist have different field areas to work based on their specialization, WMSDs in physiotherapist working in different field can also differ. further research can be done for prevalence of WMSDs in physiotherapist working in different field.

Conflict of interest: Nil.

\section{Conflict of Interest: None}

\section{REFERENCES}

1. Bindu PH and Thiruppathi A. Work related musculoskeletal discomfort among physiotherapists. International Journal of Physiotherapy. 2014;1(4):200-204. DOI: https://doi.org/10.15621/ijphy/2014/v1i4/54 558.

2. Dsouza S, Mathew A, Kumar DKU. Work related musculoskeletal disorders in physiotherapist; prevalence and associated factors: a review of literature. Int J Health Sci Res. 2016; 6(6):344-351

3. Buddhadev NP, Kotecha IS. Work-related musculoskeletal disorders: a survey of physiotherapists in Saurashtra region. National journal of medical research. 2012;2(2):178-181.

4. Adegoke BO, Akodu AK, Oyeyemi AL. Work-related musculoskeletal disorders among Nigerian physiotherapists. BMC musculoskeletal disorders. 2008 Dec;9(1):19.

5. Nordin NA, Leonard JH, Thye NC. Workrelated injuries among physiotherapists in public hospitals: a Southeast Asian picture. Clinics. 2011; 66:373-8.

6. Cromie JE, Robertson VJ, Best MO. Workrelated musculoskeletal disorders in physical therapists: prevalence, severity, risks, and responses. Physical therapy. 2000 Apr 1;80(4):336-51.

7. Ramanandi VH, Desai AR. Association of working hours, job position, and BMI with work-related musculoskeletal disorders among the physiotherapists of Gujarat-an observational study. Bulletin of Faculty of Physical Therapy. 2021 Dec;26(1):1-6.

8. Ramanandi V, Desai A. Prevalence and Risk Factors of work-related Musculoskeletal Disorders among Indian Physiotherapists: A Narrative Review of Literature. Archives of Occupational Health. 2021 Apr 10;5(2):961-8.

9. Mondal A, Mehedi MMH. Work Related Musculoskeletal Disorders among Physiotherapists in Dhaka City. Bone Muscle. 2019;2:001- 004

10. Happy SM. Common work-related musculoskeletal disorder among the physiotherapists of neurology unit at CRP (Doctoral dissertation, Bangladesh Health Professions Institute, Faculty of Medicine, the University of Dhaka, Bangladesh.).

http://hdl.handle.net/123456789/398 201608-16

11. Milhem M, Kalichman L, Ezra D, Alperovitch-Najenson D. Work-related musculoskeletal disorders among physical therapists: A comprehensive narrative review. International journal of occupational medicine and environmental health. 2016 Jul 4;29(5):735-47.

12. Sharan Deepak, PS Ajeesh. Injury prevention in physiotherapists - a scientific review Work. 2012;41:1855-1859 DOI: 10.3233/WOR-2012-0397-1855 IOS Press

13. Islam M. Common Work-Related Musculoskeletal Disorders Among the Paediatric Physiotherapists at CRP (Doctoral dissertation, Department of Physiotherapy, Bangladesh Health Professions Institute, CRP); Aug 2012

14. Anyfantis ID, Biska A. Musculoskeletal disorders among Greek physiotherapists: Traditional and emerging risk factors. Safety and health at work. 2018 Sep 1;9(3):314-8. 
15. Rugelj D. Low back pain and other workrelated musculoskeletal problems among physiotherapists. Applied ergonomics. 2003 Nov 1;34(6):635-9.

16. Maheshwari P, Soni R, Parkash N. Work related musculoskeletal disorders: a survey of physiotherapists in tricity. International Journal of Physiotherapy. 2015 Dec 1;2(6):1091-6.

17. Malarvizhi D, Kumar MK, Sivakumar VP. Prevalence of work-related musculoskeletal disorders among clinical and teaching physiotherapists-an observational study. International Journal of Physiotherapy. 2017 Apr 1;4(2):86-92.

18. Da Costa, B. R., Vieira, E. R. Risk factors for work-related musculoskeletal disorders: a systematic review of recent longitudinal studies. American Journal of Industrial Medicine, 2010;53(3): 285-323.

19. Kumar S. Theories of musculoskeletal injury causation. Ergonomics. 2001;44(1):1

20. Karanikas N, Jani BD. Frequency of examination and perceived contribution of factors relating to work-related musculoskeletal disorders of physiotherapists. International Journal of Occupational Safety and Ergonomics. 2021 Jan 19:1-8.

How to cite this article: Desai RG, Shah MK. Prevention of work-related musculoskeletal disorders in physiotherapist - a review. International Journal of Research and Review. 2021; 8(12): 497-502. DOI: https://doi.org/10. 52403/ijrr.20211261 